\title{
Formation Control of Multirobot Based on I/O Feedback Linearization and Potential Function
}

\author{
Jie Dong, Sen Liu, and Kaixiang Peng \\ Key Laboratory of Advanced Control of Iron and Steel Process of Ministry of Education, School of Automation and \\ Electrical Engineering, University of Science and Technology, Beijing 100083, China \\ Correspondence should be addressed to Kaixiang Peng; kaixiang@ustb.edu.cn
}

Received 24 January 2014; Revised 30 March 2014; Accepted 3 May 2014; Published 14 May 2014

Academic Editor: Housheng Su

Copyright (C) 2014 Jie Dong et al. This is an open access article distributed under the Creative Commons Attribution License, which permits unrestricted use, distribution, and reproduction in any medium, provided the original work is properly cited.

\begin{abstract}
Standard techniques of I/O linearization are widely applied to leader-follower approach for multirobot formation control. However general leader-follower approach cannot adapt to the environment with obstacles. Concerning that issue, a formation control method of multirobot system based on potential function is proposed in this paper, and a new control law is designed by choosing a proper potential function and employing Lyapunov stability theory, which stabilizes the formation of the multirobot system. We combine the method with a leader-follower approach to solve the problem that the latter cannot avoid obstacles. Simulation results are given to validate the method.
\end{abstract}

\section{Introduction}

Multirobot formation control is a common cooperation problem of multirobot system. In recent years, as one of the basic coordination and cooperation issues, it has been gradually applied to military reconnaissance, search, demining, aircraft formation flying, space exploration, and many other fields and attracted a large number of researchers. It has become a very active research direction of multirobot system [1]. At present, formation control methods mainly include leaderfollower approach [2-5], artificial potential field approach [6-9], behavior-based approach [10], and virtual structural approach [11]. To achieve a reasonable and efficient formation, the key is to use a proper control method that meets the system requirements. No matter what kind of control method is used, the stability of the system must be analyzed. Lyapunov stability theory $[12-14]$ and graph theory $[15,16]$ are usually used in stability analysis, and these control theory researches contribute to the improvements of multirobot system. Lyapunov method plays a very important role in a multirobot system's stability analysis and controller design. In [17], the idea of relative-position-based formation stability was proposed and the Lyapunov method was also used to design the decentralized controllers, along with an extended linear matrix inequality (LMI) to analyze the conditions required for formation stability. In [18], Lyapunov method was used to analyze the stability of multiagent coordination control. For each individual robot, there is a control Lyapunov function, and the Lyapunov function of the system is the weighted sum of all the agents' Lyapunov functions. However, that paper presents a method based on Lyapunov stability theory, which gives a stable formation control law of the multirobot system by selecting an appropriate artificial potential field function. We combine this method with $l-\varphi$ control based on inputoutput feedback linearization [19] to solve the problem that $l-\varphi$ control cannot adapt to obstacles environment.

\section{Mathematical Model}

In this section, the kinematics and dynamics of mobile robots are described in Figure 1. The robot consists of two driving rear wheels and a supporting roller, and the nonholonomic constraint that the driving wheels allow only pure rolling without slipping is satisfied. The kinematic and dynamic equations of motion of the $i$ th individual robot are given as

$$
\begin{aligned}
& \dot{x}_{i}=v_{i} \cos \theta_{i}, \\
& \dot{y}_{i}=v_{i} \sin \theta_{i},
\end{aligned}
$$




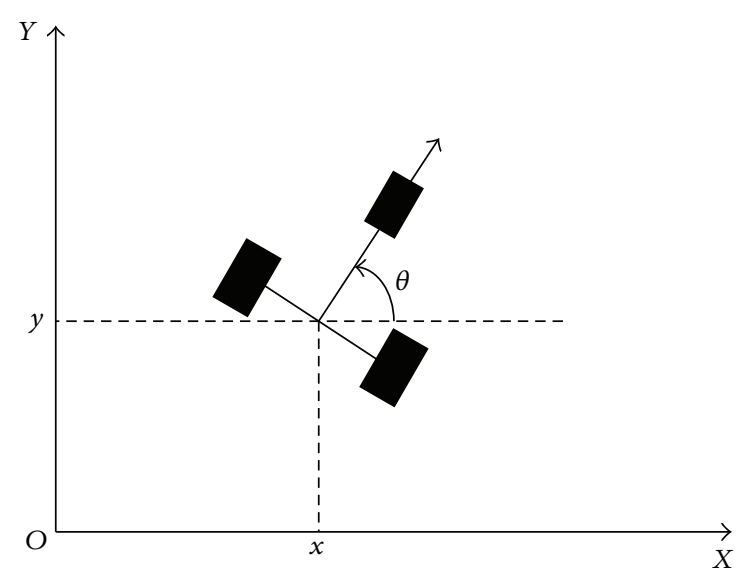

FIGURE 1: Mathematical model of the robot.

$$
\begin{aligned}
& \dot{\theta}_{i}=\omega_{i}, \\
& \dot{v}_{i}=\alpha_{i}, \\
& \dot{\omega}_{i}=\beta_{i},
\end{aligned}
$$

where the position of the robot is given by $\left(x_{i}, y_{i}\right)^{T} . \theta_{i}$ is the heading direction of the robot in the global coordinates. $q_{i}=$ $\left(x_{i}, y_{i}, \theta_{i}\right)^{T}$ is the pose vector of robot $i . v_{i}$ and $\omega_{i}$ are the linear and angular velocities at the center of the axle of each robot. $u_{i}=\left(\alpha_{i}, \beta_{i}\right)^{T}$ is control input (acceleration).

\section{Control of Formations}

3.1. I- $\varphi$ Control Based on Input-Output Feedback Linearization. $l-\varphi$ control is a kind of leader-follower approach. A group of two nonholonomic mobile robots is shown in Figure 2. The aim of the controller is to make the follower (Robot2) follow the leader (Robot1) by keeping the relative distance $l_{12}$ and the relative orientation $\varphi_{12}$ constant. As a result, both the values can be controlled to reach the desired value $\left(l_{12}^{d}, \varphi_{12}^{d}\right)$ and maintain the desired formation.

We introduce $\gamma_{1}=\theta_{1}+\varphi_{12}-\theta_{2}$; according to the geometrical relationship, the differential equation of $l_{12}$ and $\varphi_{12}$ is given by

$$
\begin{aligned}
& \dot{l}_{12}=v_{2} \cos \gamma_{1}-v_{1} \cos \varphi_{12}+d \omega_{2} \sin \gamma_{1}, \\
& \dot{\varphi}_{12}=\frac{1}{l_{12}}\left(v_{1} \sin \varphi_{12}-v_{2} \sin \gamma_{1}+d \omega_{2} \cos \gamma_{1}-l_{12} \omega_{1}\right), \\
& \dot{\theta}_{12}=\omega_{2},
\end{aligned}
$$

where $v_{i}$ and $\omega_{i}(i=1,2)$ are the linear and angular velocities at the center of the axle of each robot. The distance between the supporting roller and the center of the axis of the driving wheels of each robot is denoted by $d$. In order to avoid collisions between robots, we will require that $l_{12}>d$.

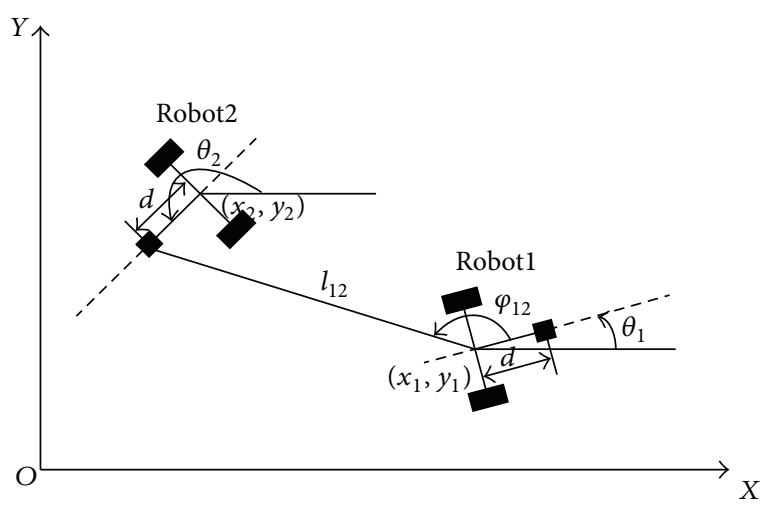

FIGURE 2: Notation for $l-\varphi$ control.

We use standard techniques of $\mathrm{I} / \mathrm{O}$ linearization to generate a control law [12], which is given by

$$
\begin{aligned}
& \omega_{2}=\frac{\cos \gamma_{1}}{d}\left\{a_{2} l_{12}\left(\varphi_{12}^{d}-\varphi_{12}\right)\right. \\
& \left.\quad-v_{1} \sin \varphi_{12}+l_{12} \omega_{1}+\rho_{12} \sin \gamma_{1}\right\}, \\
& v_{2}=\rho_{12}-d \omega_{2} \tan \gamma_{1},
\end{aligned}
$$

where

$$
\rho_{12}=\frac{a_{1}\left(l_{12}^{d}-l_{12}\right)+v_{1} \cos \varphi_{12}}{\cos \gamma_{1}} .
$$

That leads to dynamics in the $l-\varphi$ variables of the form

$$
\begin{gathered}
\dot{l}_{12}=a_{1}\left(l_{12}^{d}-l_{12}\right), \\
\dot{\varphi}_{12}=a_{2}\left(\varphi_{12}^{d}-\varphi_{12}\right),
\end{gathered}
$$

where $a_{1}$ and $a_{2}$ are constants. By selecting appropriate values, the relative distance and angle between the robots can converge to $\left(l_{12}^{d}, \varphi_{12}^{d}\right)$, and the system becomes stable.

3.2. Control Based on Artificial Potential Field Function. An artificial potential field approach is a virtual force method proposed by Khatib. Its basic idea is that the robots move in a virtual force field. Obstacles are surrounded by repulsive potential field, which produces a repulsive force that decreases rapidly with the closer distance between the robot and the obstacle. The target is surrounded by attractive potential field, which produces an attractive force that decreases as the robot is close to the target. Under the role of the resultant force, the robots move along the direction of minimizing potential energy. For multirobot formation control, we need to design a suitable potential function to maintain the formation while reaching the target and avoiding obstacles. Potential function is defined as follows.

Definition 1 (potential function). Potential function $V_{i j}$ is a differentiable, nonnegative, and unbounded function of 


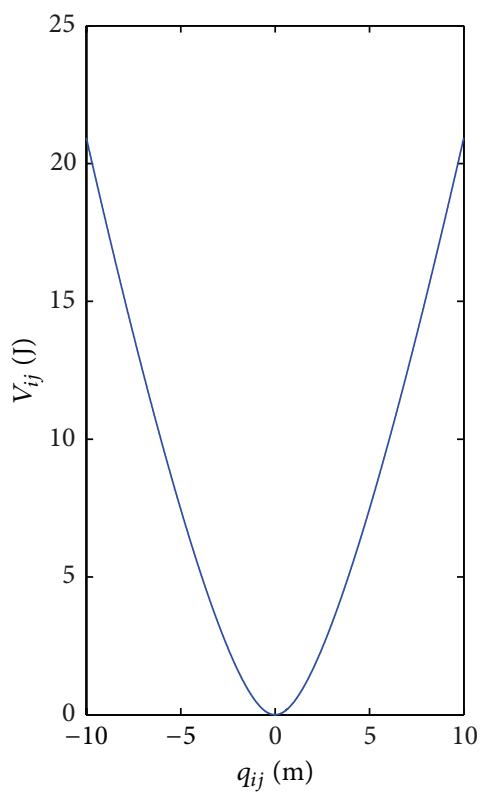

(a)

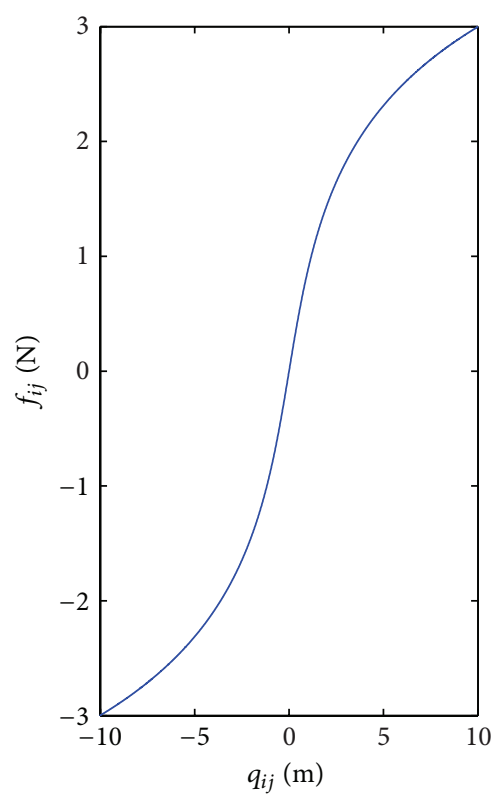

(b)

Figure 3: Potential energy and force.

the distance $\left\|r_{i j}\right\|$ between robot $i$ and $j$, and the following conditions are satisfied.

(1) When $\left\|r_{i j}\right\| \rightarrow \infty, V_{i j}\left(\left\|r_{i j}\right\|\right) \rightarrow \infty$.

(2) When the distance $\left\|r_{i j}\right\|$ achieves the desired distance, that is, $\left\|r_{i j}\right\|=d_{i j}, V_{i j}$ gets the only minimum value.

The potential function of neighbor robot $i$ and $j$ is chosen as follows:

$$
V_{i j}\left(q_{i j}\right)=K_{i j}\left(q_{i j} \ln \left(q_{i j}+\sqrt{q_{i j}^{2}+1}\right)-\sqrt{q_{i j}^{2}+1}\right)+1
$$

where $q_{i j}=\left\|r_{i j}\right\|-d_{i j}, K_{i j}$ is a positive constant, and then the force is the negative gradient of the potential energy:

$$
f_{i j}\left(r_{i}, r_{j}\right)=-\operatorname{grad}\left(V_{i j}\right)=K_{i j} \operatorname{arsh}\left(q_{i j}\right) \frac{r_{j}-r_{i}}{\left\|r_{i j}\right\|},
$$

where $r_{i}, r_{j}$ are the position vectors of robots $i$ and $j$.

The potential energy $V_{i j}$ and force $f_{i j}$ described by (6) and (7) are shown in Figure 3. It indicates that when $q_{i j}>0$, that is, $\left\|r_{i j}\right\|>d_{i j}, f_{i j}>0$, the robots are attractive, when $q_{i j}<0$, that is, $\left\|r_{i j}\right\|<d_{i j}, f_{i j}<0$, the robots are repulsive, and when $q_{i j}=0$, that is, $\left\|r_{i j}\right\|=d_{i j}, f_{i j}=0$, the force between the robots achieves balance, and $V_{i j}$ is minimized.

\section{Control Based on I/O Feedback Linearization and Potential Function}

4.1. Design of a Stable Formation Control Law. Considering a system of $\mathrm{n}$ robots, the dynamic of each robot is described by (1). The total potential energy and force acting on robot $i$ contributed from all the other robots are given as

$$
\begin{gathered}
V_{i}\left(r_{i}\right)=\sum_{j \neq i} V_{i j}\left(q_{i j}\right), \\
F_{i}\left(r_{i}\right)=\sum_{j \neq i} f_{i j}\left(r_{i}, r_{j}\right) .
\end{gathered}
$$

When the formation moves toward the target in an environment with obstacles, $F_{i}\left(r_{i}\right)$ also includes the attractive force of the target and the repulsive force of the obstacles. The magnitude of $F_{i}\left(r_{i}\right)$ projected in the heading direction of the robot is used to control the translational acceleration of the robot. The angle is used as the desired orientation for the heading direction of the robot. Let $\phi_{i}$ be the direction of the resultant force of robot $i$, and the orientation error can be described as

$$
\begin{aligned}
& e_{i}=\theta_{i}-\phi_{i}, \\
& \dot{e}_{i}=\dot{\theta}_{i}-\dot{\phi}_{i}, \\
& \ddot{e}_{i}=\ddot{\theta}_{i}-\ddot{\phi}_{i} .
\end{aligned}
$$

Theorem 2. For a multirobot system described by (1), the potential force shown by (9) acts on each robot, and the angle of the resultant force is defined as the desired orientation of them. Then, the following control law stabilizes the formation of the system:

$$
\begin{gathered}
\alpha_{i}=-\sqrt{f_{i x}^{2}+f_{i y}^{2}}-k_{1} v_{i}, \\
\beta_{i}=-k_{2}\left(\theta_{i}-\phi_{i}\right)-k_{3}\left(\dot{\theta}_{i}-\dot{\phi}_{i}\right)+\ddot{\phi}_{i},
\end{gathered}
$$


where $k_{1}, k_{2}, k_{3}$ are positive constants and $f_{i x}, f_{i y}$ are the components of $F_{i}\left(r_{i}\right)$ in the $x$ and $y$ directions, respectively.

Proof. (1) $v_{i} \geq 0$. Consider the following Lyapunov candidate:

$$
W=\sum_{i=1}^{n} V_{i}+\frac{1}{2} \sum_{i=1}^{n} k_{2} e_{i}^{2}+\frac{1}{2} \sum_{i=1}^{n} \dot{e}_{i}^{2}+\frac{1}{2} \sum_{i=1}^{n} v_{i}^{2} .
$$

The time derivative of $W$ is computed as

$$
\dot{W}=\sum_{i=1}^{n}\left\{\left(\nabla_{r_{i}} V_{i}\right)^{T} \dot{r}_{i}+k_{2} e_{i} \dot{e}_{i}+\dot{e}_{i} \ddot{e}_{i}+v_{i} \dot{v}_{i}\right\} .
$$

Insert (11) into (13):

$$
\begin{gathered}
\dot{W}=\sum_{i=1}^{n}\left\{v_{i} f_{i x} \cos \theta_{i}+v_{i} f_{i y} \sin \theta_{i}\right. \\
\left.\quad-k_{3} \dot{e}_{i}^{2}-v_{i} \sqrt{f_{i x}^{2}+f_{i y}^{2}}-k_{1} v_{i}^{2}\right\} \\
=\sum_{i=1}^{n}\left\{v_{i}\left|F_{i}\right|\left(\cos \left(\theta_{i}-\phi_{i}\right)-1\right)-k_{3} \dot{e}_{i}^{2}-k_{1} v_{i}^{2}\right\},
\end{gathered}
$$

where $\left|F_{i}\right|=\sqrt{f_{i x}^{2}+f_{i y}^{2}}$, due to $\cos \left(\theta_{i}-\phi_{i}\right) \leq 1$, so $\dot{W} \leq 0$.

(2) $v_{i}<0$. Consider the following Lyapunov candidate:

$$
W=2 \sum_{i=1}^{n} V_{i}+\frac{1}{2} \sum_{i=1}^{n} k_{2} e_{i}^{2}+\frac{1}{2} \sum_{i=1}^{n} \dot{e}_{i}^{2}+\frac{1}{2} \sum_{i=1}^{n} v_{i}^{2} .
$$

Insert (11) into (15), and then calculate the derivative of $W$ :

$$
\dot{W}=\sum_{i=1}^{n}\left\{v_{i}\left|F_{i}\right|\left(2 \cos \left(\theta_{i}-\phi_{i}\right)-1\right)-k_{3} \dot{e}_{i}^{2}-k_{1} v_{i}^{2}\right\} \text {. }
$$

From (10),

$$
\begin{aligned}
e_{i}=\left\{c_{1} \cos \left(\frac{\sqrt{4 k_{2}-k_{3}^{2}}}{2} t\right)\right. \\
\left.+c_{2} \sin \left(\frac{\sqrt{4 k_{2}-k_{3}^{2}}}{2} t\right)\right\} e^{-\left(k_{3} / 2\right) t}
\end{aligned}
$$

Let $c_{1}=c_{2}=0.5$, and then $\left|e_{i}\right|<1$, that is, $2 \cos \left(\theta_{i}-\phi_{i}\right)-$ $1=2 \cos e_{i}-1>0$, so $\dot{W} \leq 0$.

Consequently, the stability of the formation is proved with Lyapunov stability theory.

4.2. Combination Control Method. $l-\varphi$ control based on $\mathrm{I} / \mathrm{O}$ feedback linearization is simple, that we just control the follower robot to follow the trajectory of the leader robot. In this way, the formation control can be simplified to an independent tracking problem. Each robot only needs to obtain the status information of its leader, which greatly sim-

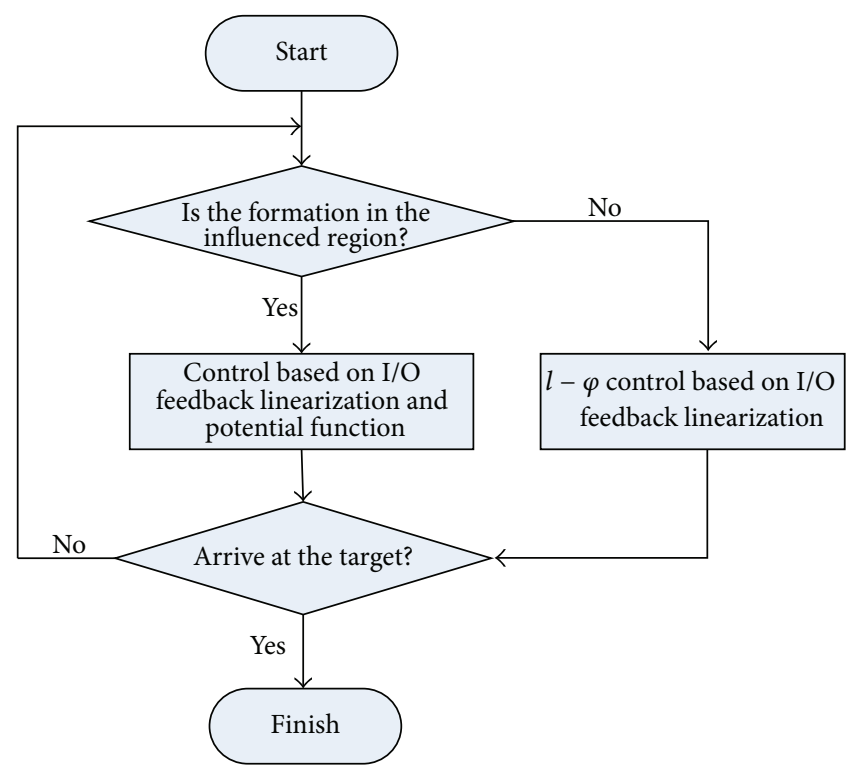

FIGURE 4: The algorithm flow chart of combination method.

plifies the issue of cooperation among robots. However, this feedback control cannot avoid obstacles, while the artificial potential field approach is effective in obstacle avoidance. For this reason, if we combine those two methods, the problem will be solved.

The strategy is as follows. The leader adopts the artificial potential field approach for path planning to reach the target while avoiding obstacles. The follower uses I/O feedback linearization combination with potential function to maintain formation in the obstacles environment; that is, when the follower is in the region influenced by obstacles, the combination method is applied, and the control performance can be improved by adjusting the weight, and then when out of the influenced region, $l-\varphi$ control based on I/O feedback linearization is only used. The algorithm flow chart is shown in Figure 4.

\section{Simulation}

In this section, computer simulation is used to verify the previous conclusions. For a group of 3 robots, the robots maintain triangular formation to reach the target while avoiding obstacles, which were idealized as circular objects just as described in [20].

(1) Formation Control Based on Potential Function. The initial positions of the robots are $\left[\begin{array}{ll}-2 & 0\end{array}\right],\left[\begin{array}{ll}0 & 3\end{array}\right]$, and [ $\left[\begin{array}{ll}0 & 0\end{array}\right]$. The position of the target is $\left[\begin{array}{ll}25 & 20\end{array}\right]$. The obstacle is a circle of radius 1 centered at [ 79 9. Under the role of the potential energy described by (6) and applying the control law (11), the robots form an equilateral triangle formation with the side length of 3 , avoid the obstacle, keep the same speed and direction, and reach the target at last just as shown in Figure 5. Figure 6 illustrates the change of errors $e_{i}$. It is observed that the formation is stable. 


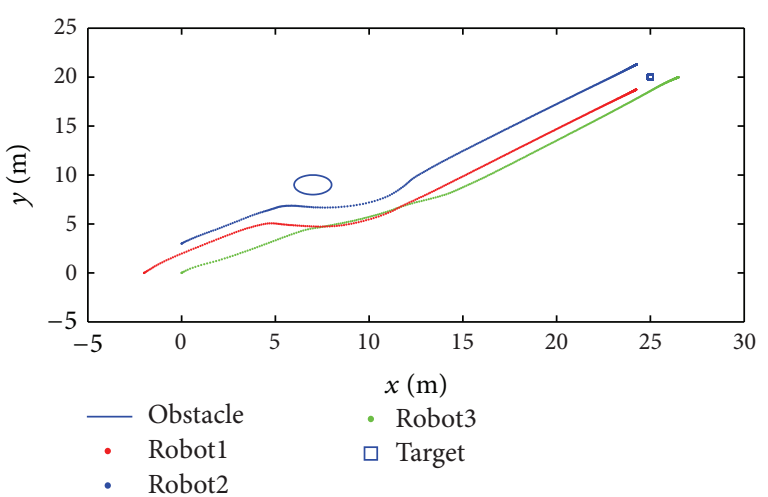

FIGURE 5: Formation control based on potential function.

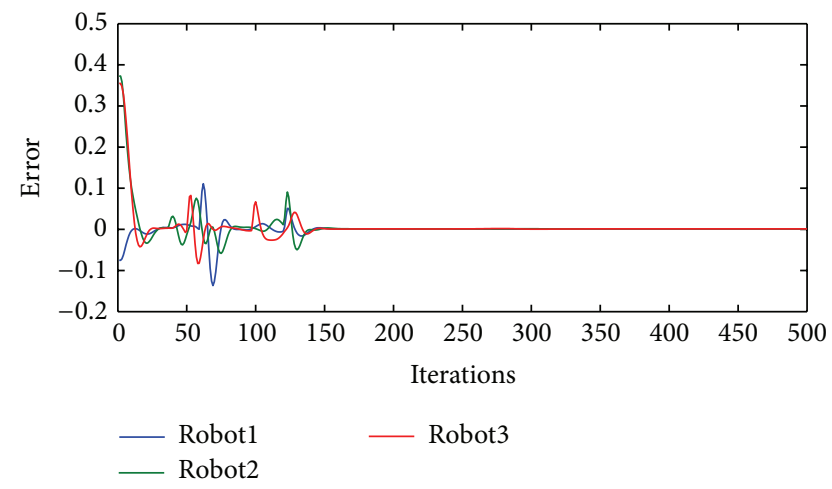

FIGURE 6: The orientation error of robots.

(2) Control Based on $l-\varphi$ and Potential Function. The initial positions of the robots are $\left[\begin{array}{ll}0 & 1\end{array}\right],\left[\begin{array}{ll}-3 & -2\end{array}\right]$, and $\left[\begin{array}{ll}-2 & 0\end{array}\right]$. The position of the target is $\left[\begin{array}{ll}20 & 16\end{array}\right]$. The obstacle is a circle of radius 1 centered at [ $\left.\begin{array}{ll}9 & 7\end{array}\right]$. Robotl is the leader, and Robot2, Robot 3 are the followers keeping a distance of 2 , angles of $\pi / 3$, $-\pi / 3$, respectively, from Robot1. When the formation enters into the influenced region of the obstacle, use the approach of control based on $l-\varphi$ and potential function. Otherwise, $l-\varphi$ control based on I/O feedback linearization is applied. Figure 7 illustrates that single $l-\varphi$ control cannot avoid obstacles, while by using the proposed combination control method, the formation can successfully round the obstacle and move to the target keeping a stable formation, as shown in Figure 8.

\section{Conclusions}

Formation control of multirobot system was studied in the paper. A new artificial potential field function and corresponding formation controller were designed, and the stability analysis was given. In addition, we proposed a combination control method based on $\mathrm{I} / \mathrm{O}$ feedback linearization and potential function, of which feature is choosing the appropriate control strategy according to obstacles environment, solving the problem that $l-\varphi$ control cannot avoid obstacles.

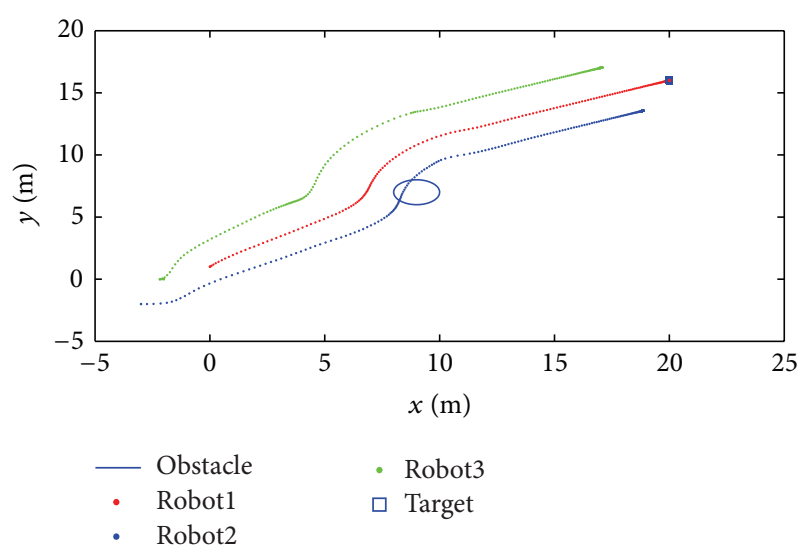

FIGURE 7: $l-\varphi$ control cannot avoid obstacles.

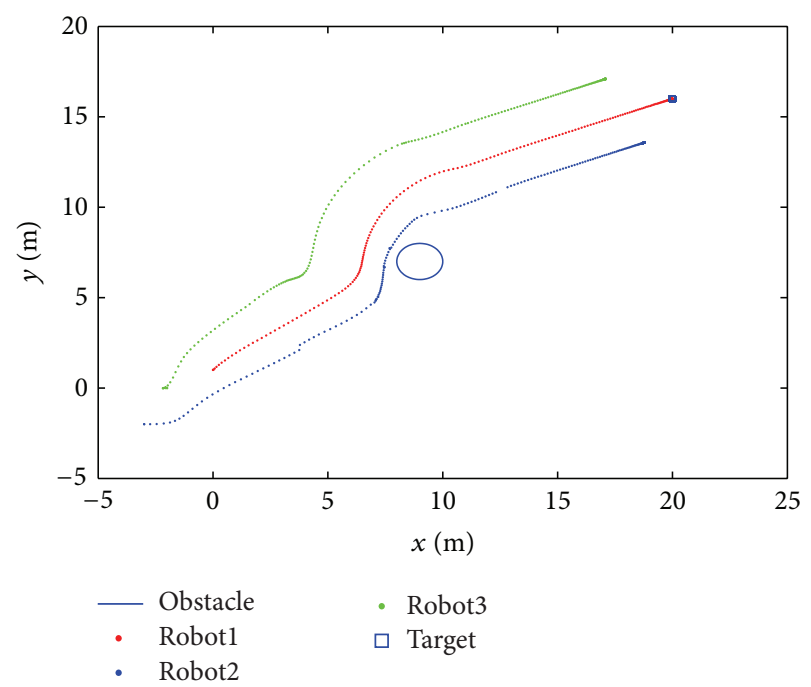

FIGURE 8: Control based on $l-\varphi$ and potential function.

\section{Conflict of Interests}

The authors declare that there is no conflict of interests regarding the publication of this paper.

\section{Acknowledgments}

This work was supported by the Natural Science Foundation of China (NSFC) under Grant no. 61074085 and by Beijing Natural Science Foundation (4142035), China.

\section{References}

[1] L. Lei, Formation and Coordination Control of Multiple Mobile Robots, Huazhong University of Science and Technology, Wuhan, China, 2009.

[2] X. Chen, A. Serrani, and H. Ozbay, "Control of leader-follower formations of terrestrial UAVs," in Proceedings of the 42nd IEEE Conference on Decision and Control, vol. 1, pp. 498-503, December 2003. 
[3] K. H. Kowdiki, R. K. Barai, and S. Bhattacharya, "Leaderfollower formation control using artificial potential function: a kinematic approach," in Proceeding of the IEEE-International Conference on Advances in Engineering, Science and Management, pp. 500-505, Tamil Nadu, India, March 2012.

[4] H. Su, X. Wang, and Z. Lin, "Flocking of multi-agents with a virtual leader," IEEE Transactions on Automatic Control, vol. 54, no. 2, pp. 293-307, 2009.

[5] H. Su, X. Wang, and G. Chen, "A connectivity-preserving flocking algorithm for multi-agent systems based only on position measurements," International Journal of Control, vol. 82, no. 7, pp. 1334-1343, 2009.

[6] O. Khatib, "Real-time obstacle avoidance for manipulators and mobile robots," The International Journal of Robotics Research, vol. 5, no. 1, pp. 90-98, 1986.

[7] J. Sfeir, M. Saad, and H. Saliah-Hassane, "An improved artificial potential field approach to real-time mobile robot path planning in an unknown environment," in Proceeding of the 9th IEEE International Symposium on Robotic and Sensors Environments, pp. 208-213, Montreal, Canada, September 2011.

[8] R. Olfati-Saber and R. M. Murray, "Distributed cooperative control of multiple vehicle formations using structural potential functions," in Proceeding of the IFAC World Congress, pp. 346352, 2002.

[9] R. Olfati-Saber, "Flocking with obstacle avoidance," Tech. Rep. CIT-CDS03-006, California Institute of Technology, Control and Dynamical Systems, Pasadena, Calif, USA, 2003.

[10] T. Balch and R. C. Arkin, "Behavior-based formation control for multirobot teams," IEEE Transactions on Robotics and Automation, vol. 14, no. 6, pp. 926-939, 1998.

[11] W. Ren and R. W. Beard, "Decentralized scheme for spacecraft formation flying via the virtual structure approach," Journal of Guidance, Control and Dynamics, vol. 27, no. 1, pp. 73-82, 2004.

[12] H. Su, N. Zhang, M. Z. Q. Chen, H. Wang, and X. Wang, "Adaptive flocking with a virtual leader of multiple agents governed by locally Lipschitz nonlinearity," Nonlinear Analysis: Real World Applications, vol. 14, no. 1, pp. 798-806, 2013.

[13] A. Yang, W. Naeem, G. W. Irwin, and K. Li, "Stability analysis and implementation of a decentralized formation control strategy for unmanned vehicles technology," IEEE Transactions on Control Systems Technology, vol. 22, no. 2, pp. 706-720, 2014.

[14] X. Luo, N. Han, and X. Guan, "Leader-following consensus protocols for formation control of multi-agent network," Journal of Systems Engineering and Electronics, vol. 22, no. 6, pp. 991-997, 2011.

[15] J. A. Fax and R. M. Murray, "Information flow and cooperative control of vehicle formations," IEEE Transactions on Automatic Control, vol. 49, no. 9, pp. 1465-1476, 2004.

[16] M. M. Zavlanos, M. B. Egerstedt, and G. J. Pappas, "Graphtheoretic connectivity control of mobile robot networks," Proceedings of the IEEE, vol. 99, no. 9, pp. 1525-1540, 2011.

[17] D. Xue, J. Yao, G. Chen, and Y. L. Yu, "Formation control of networked multi-agent systems," IET Control Theory and Applications, vol. 4, no. 10, pp. 2168-2176, 2010.

[18] P. Ogren, M. Egerstedt, and X. Hu, "A control Lyapunov function approach to multiagent coordination," IEEE Transactions on Robotics and Automation, vol. 18, no. 5, pp. 847-851, 2002.

[19] J. P. Desai, J. Ostrowski, and V. Kumar, "Controlling formations of multiple mobile robots," in Proceedings of the IEEE International Conference on Robotics and Automation, vol. 4, pp. 28642869, Leuven, Belgium, May 1998.
[20] Y. Liang and H. Lee, "Decentralized formation control and obstacle avoidance for multiple robots with nonholonomic constraints," in Proceedings of the American Control Conference, pp. 5596-5601, Minneapolis, Minn, USA, June 2006. 


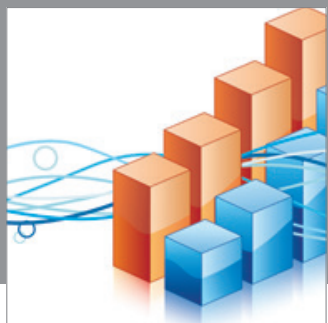

Advances in

Operations Research

mansans

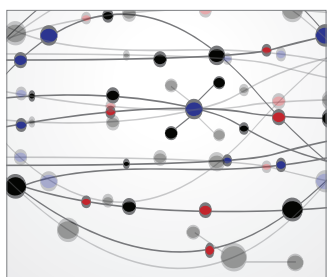

The Scientific World Journal
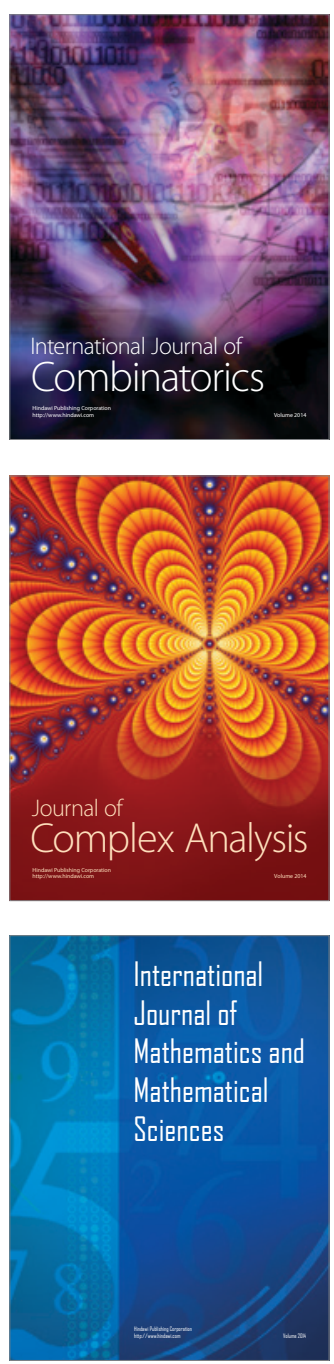
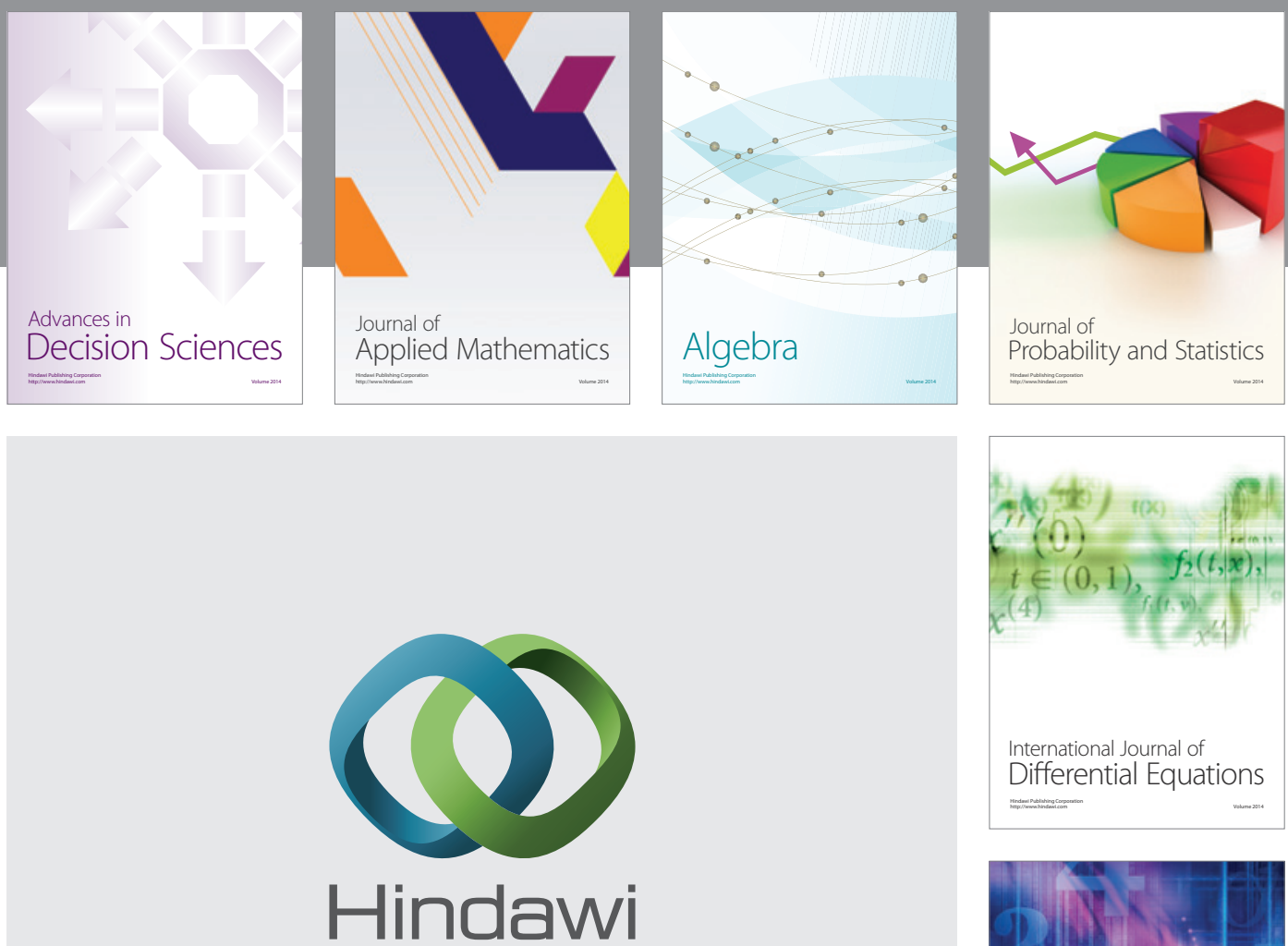

Submit your manuscripts at http://www.hindawi.com
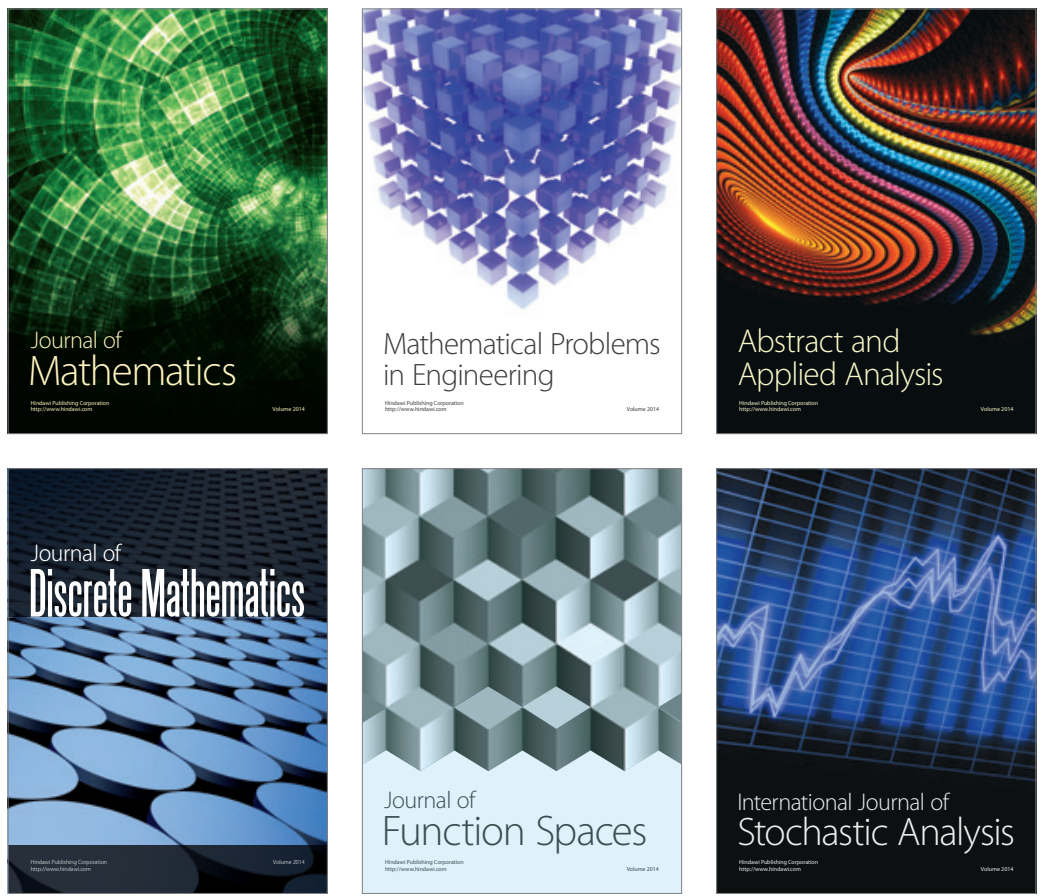

Journal of

Function Spaces

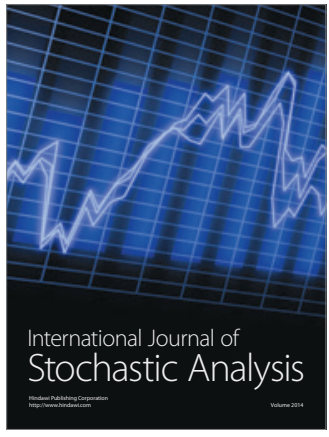

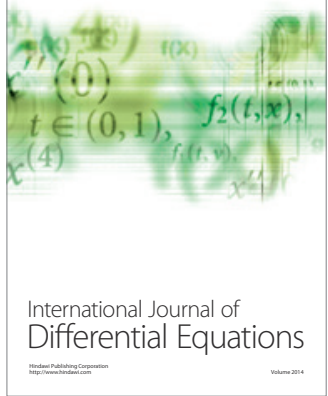
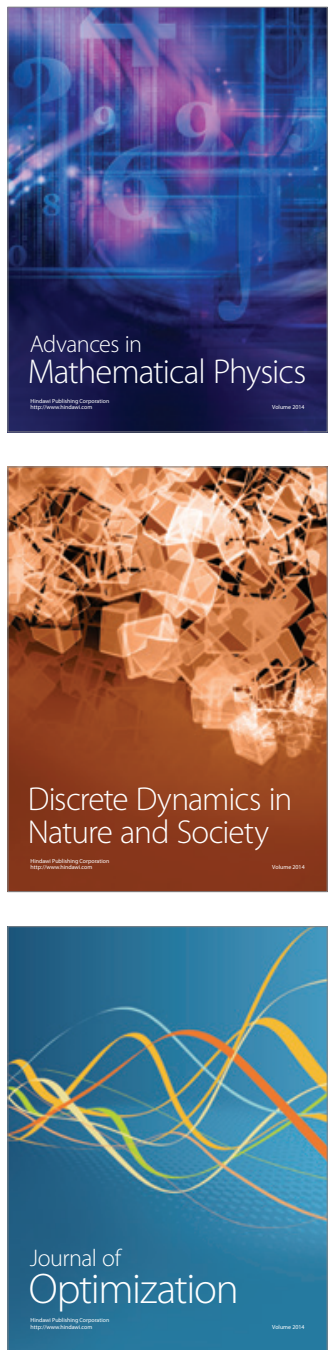